\title{
Using geographic information systems to identify prospective marketing areas for a special library Rozalynd P McConnaughy* ${ }^{*}$ and Steven P Wilson ${ }^{\dagger}$
}

\author{
Address: School of Medicine Library, University of South Carolina, 6311 Garners Ferry Road, Columbia, SC 29208, USA \\ Email: Rozalynd P McConnaughy* - roz@gw.med.sc.edu; Steven P Wilson - wilsons@gw.med.sc.edu \\ * Corresponding author †Equal contributors
}

Published: 04 May 2006

Biomedical Digital Libraries2006, 3:4 doi:10.1 186/1742-558I-3-4

This article is available from: http://www.bio-diglib.com/content/3/l/4

(C) 2006McConnaughy and Wilson; licensee BioMed Central Ltd.

This is an Open Access article distributed under the terms of the Creative Commons Attribution License (http://creativecommons.org/licenses/by/2.0), which permits unrestricted use, distribution, and reproduction in any medium, provided the original work is properly cited.
Received: 02 February 2006

Accepted: 04 May 2006

\begin{abstract}
Background: The Center for Disability Resources (CDR) Library is the largest collection of its kind in the Southeastern United States, consisting of over 5,200 books, videos/DVDs, brochures, and audiotapes covering a variety of disability-related topics, from autism to transition resources. The purpose of the library is to support the information needs of families, faculty, students, staff, and other professionals in South Carolina working with individuals with disabilities. The CDR Library is funded on a yearly basis; therefore, maintaining high usage is crucial. A variety of promotional efforts have been used to attract new patrons to the library. Anyone in South Carolina can check out materials from the library, and most of the patrons use the library remotely by requesting materials, which are then mailed to them. The goal of this project was to identify areas of low geographic usage as a means of identifying locations for future library marketing efforts.
\end{abstract}

Methods: Nearly four years worth of library statistics were compiled in a spreadsheet that provided information per county on the number of checkouts, the number of renewals, and the population. Five maps were created using ArcView GIS software to create visual representations of patron checkout and renewal behavior per county.

Results: Out of the 46 counties in South Carolina, eight counties never checked out materials from the library. As expected urban areas and counties near the library's physical location have high usage totals.

Conclusion: The visual representation of the data made identification of low usage regions easier than using a standalone database with no visual-spatial component. The low usage counties will be the focus of future Center for Disability Resources Library marketing efforts. Due to the impressive visual-spatial representations created with Geographic Information Systems, which more efficiently communicate information than stand-alone database information can, librarians may benefit from the software's use as a supplemental tool for tracking library usage and planning promotional efforts.

\section{Background}

The Center for Disability Resources Library (CDR Library) is a special library that serves anyone living in South Caro- lina, especially professionals who work with individuals with disabilities and family members of children with special needs. The CDR Library is a collaborative effort 
Table I: CENTER FOR DISABILITY RESOURCES LIBRARY USAGE STATISTICS:JUNE I, 200 I - FEBRUARY I7, 2005

\begin{tabular}{|c|c|c|c|c|}
\hline COUNTY & TOTAL PATRONS* & TOTAL CHECKOUT & TOTAL RENEWAL & CENSUS 2000 \\
\hline ABBEVILLE & 1 & 4 & 1 & 26,167 \\
\hline AIKEN & 13 & 240 & 145 & 142,552 \\
\hline ALLENDALE & 0 & 0 & 0 & ||$, 2||$ \\
\hline ANDERSON & 17 & 81 & 52 & 165,740 \\
\hline BAMBERG & 1 & 3 & 2 & 16,658 \\
\hline BARNWELL & 0 & 0 & 0 & 23,478 \\
\hline BEAUFORT & 3 & 10 & 1 & 120,937 \\
\hline BERKELEY & 2 & 11 & 1 & $|42,65|$ \\
\hline CALHOUN & 2 & 5 & 2 & 15,185 \\
\hline CHARLESTON & 19 & 77 & 24 & 309,969 \\
\hline CHEROKEE & 1 & 6 & 1 & 52,537 \\
\hline CHESTER & 3 & 9 & 1 & 34,068 \\
\hline CHESTERFIELD & 1 & 2 & 2 & 42,768 \\
\hline CLARENDON & 0 & 0 & 0 & 32,502 \\
\hline COLLETON & I & 4 & 4 & 38,264 \\
\hline DARLINGTON & 2 & 14 & 4 & 67,394 \\
\hline DILLON & 0 & 0 & 0 & 30,722 \\
\hline DORCHESTER & 3 & 8 & 7 & 96,413 \\
\hline EDGEFIELD & 0 & 0 & 0 & 24,595 \\
\hline FAIRFIELD & 2 & 14 & I & 23,454 \\
\hline FLORENCE & 4 & 13 & 10 & $|25,76|$ \\
\hline GEORGETOWN & 3 & 17 & 7 & 55,797 \\
\hline GREENVILLE & 13 & 65 & 29 & 379,616 \\
\hline GREENWOOD & 5 & 68 & 32 & 66,271 \\
\hline HAMPTON & 1 & 1 & 1 & 21,386 \\
\hline HORRY & 7 & 66 & 49 & 196,629 \\
\hline JASPER & 0 & 0 & 0 & 20,678 \\
\hline KERSHAW & 4 & 14 & 4 & 52,647 \\
\hline LANCASTER & 3 & 9 & 5 & 61,351 \\
\hline LAURENS & 1 & 20 & 0 & 69,567 \\
\hline LEE & 1 & 2 & 6 & 20,119 \\
\hline LEXINGTON & 21 & 92 & 46 & 216,014 \\
\hline MARION & 1 & 1 & 0 & 35,466 \\
\hline MARLBORO & 0 & 0 & 0 & 28,818 \\
\hline MCCORMICK & 0 & 0 & 0 & 9,958 \\
\hline NEWBERRY & 2 & 5 & 9 & 36,108 \\
\hline OCONEE & 5 & 14 & 5 & 66,215 \\
\hline ORANGEBURG & 2 & 4 & 0 & 91,582 \\
\hline PICKENS & 4 & 18 & 11 & 110,757 \\
\hline RICHLAND & 116 & 959 & 883 & 320,677 \\
\hline SALUDA & 4 & 19 & 18 & 19,181 \\
\hline SPARTANBURG & 8 & 18 & 11 & 253,791 \\
\hline SUMTER & 2 & 81 & 47 & 104,646 \\
\hline UNION & 1 & 7 & 1 & 29,881 \\
\hline WILLIAMSBURG & 2 & 10 & 11 & 37,217 \\
\hline YORK & 11 & 82 & 18 & $164,6 \mid 4$ \\
\hline
\end{tabular}

\footnotetext{
* Total Patrons does not include patrons that have only used the library's web resources.
}

between BabyNet/South Carolina Department of Health and Environmental Control, the Center for Disability Resources, the South Carolina Department of Disabilities and Special Needs, and the University of South Carolina School of Medicine Library. The CDR Library consists of over 5,200 books, videos, brochures, and audiotapes covering a variety of disability-related topics. Since 2001, the CDR Library has been part of the University of South Carolina School of Medicine Library, which is located in
Columbia, South Carolina. The library is funded by a yearly contract; thus, the library's utilization is vital to its existence.

Approximately 651,000 people in South Carolina have a disability [1]. An estimated 108,000 people in South Carolina have difficulty performing self-care activities, such as dressing, bathing, or other activities of daily living. Over 233,000 South Carolinians have a cognitive disabil- 


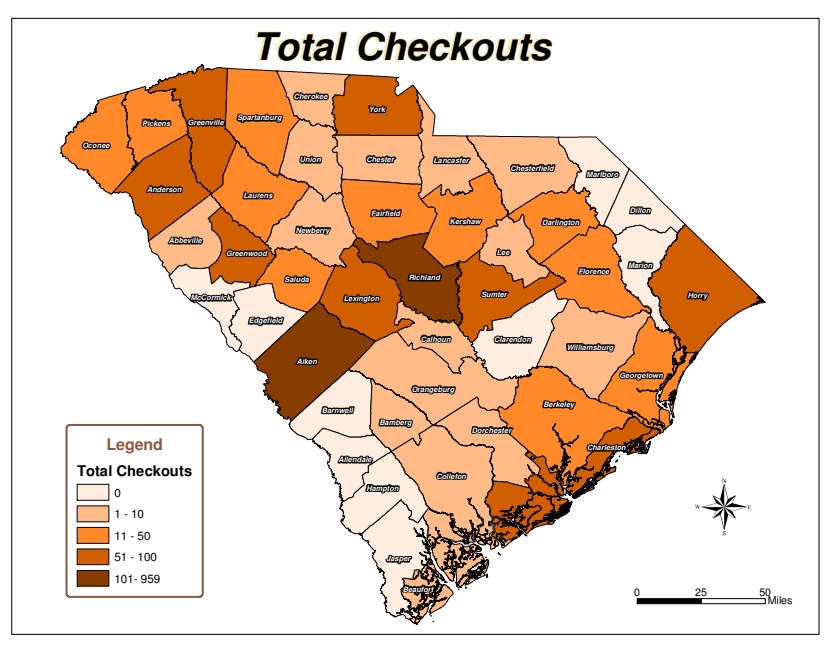

Figure I

Total Checkouts.

ity. Most of the information requests received by the CDR Library are related to mental disabilities and children. During the 2001-2002 school year, 110,037 children ages 3 to 21 in South Carolina were served under the Individuals with Disabilities Education Act (IDEA), Part B [2].

The librarians working with the CDR collection have attempted to promote the library and its services in a variety of ways. A monthly newsletter is distributed to patrons via mail and/or email to increase awareness of new resources and to promote the CDR librarians' monthly outreach activities. Librarians also give presentations and tours of the CDR Library to local support groups, University of South Carolina classes, and organizations supporting individuals with disabilities. Other marketing efforts include distributing CDR Library pamphlets by mail, advertising the library in various organizations' newsletters, and participating in live radio spots promoting the collection. The major focus of these efforts in the past has involved promoting the collection to particular groups by exhibiting at various professional conferences instead of publicizing library services to specific areas of the state. Myrtle Beach (Horry County), Charleston (Charleston County), Columbia (Richland County), and Greenville (Greenville County) are common locations for these conferences.

Geographic Information Systems (GIS) can be a very useful problem solving, planning, and service development tool for libraries. GIS has been used by libraries to study community demographics for collection development purposes. One study used demographic data that was visually represented to determine whether to add a consumer health collection in a public library [3]. Librarians have also used GIS to determine the location of new branches by plotting current library locations. In addition to having a visual representation of current library locations, Weber County Library System in northern Utah used GIS to plan the location of a new branch by analyzing patron addresses and demographic data [4]. GIS has also been used to study library book usage with regard to bookshelf heights and spatial distributions of the books [5].

The objective of this study was to identify prime locations for future library promotion efforts by determining which counties in South Carolina were utilizing the Center for Disability Resources Library the least.

\section{Methods}

Nearly four years worth of circulation statistics from June 1, 2001, to February 17, 2005, were compiled by running a report in Innovative Interfaces, the library's integrated library system. Circulation statistics obtained included the number of items checked out and renewed per patron. This information was added to an Excel spreadsheet (Table 1).

Next, each patron's address was matched to a South Carolina county by using the United States Postal Service Zip Code Lookup tool, which is freely available online [6]. Although the zip code for each patron is included in the address field of his/her patron record, the county is not included. Therefore the zip code lookup tool was used to find out which zip code corresponded to each patron's county. After entering an address and selecting mailing industry information, the USPS web site lists information, including the county of the address. Patron names and addresses were removed from the spreadsheet for confidentiality and replaced by a county name. Population

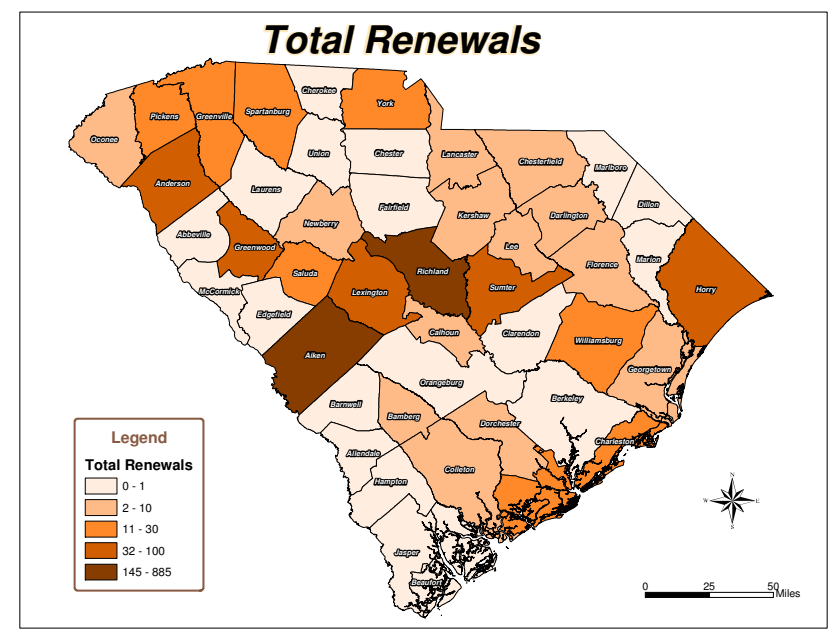

Figure 2

Total Renewals. 


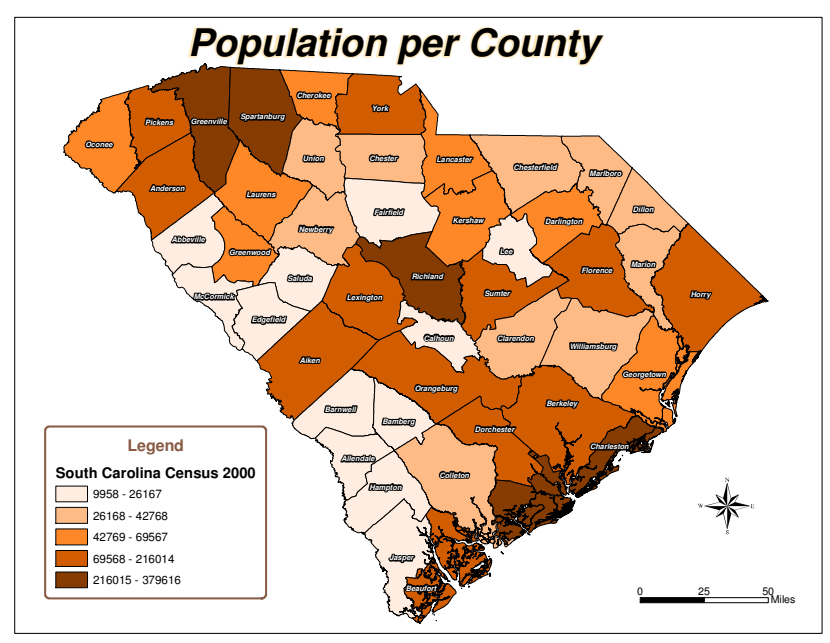

Figure 3

Population Per County.

data per county from Census 2000 data was also added to the spreadsheet [7]. The population totals were used to calculate the number of items checked out and renewed per

person.

These usage trends were spatially referenced and displayed visually using ArcView GIS (Geographic Information Systems) software. The spreadsheet data was merged with an existing shape file database containing South Carolina county location data.

Five South Carolina county maps were created: Total Checkouts per County (Figure 1), Total Renewals per County (Figure 2), Population per County (Figure 3), Checkouts per 100,000 people (Figure 4), and Renewals per 100,000 people (Figure 5).

\section{Results}

As expected, urban counties, or those nearest to major metropolitan areas like Aiken, Charleston, Greenville and Richland, and counties closest to the library's physical location had high usage totals. There are 46 counties in South Carolina. The following ten counties had the highest number of items checked out from the library: Aiken, Anderson, Charleston, Greenville, Greenwood, Horry, Lexington, Richland, Sumter, and York (Figure 1). Six counties, including Aiken, Fairfield, Greenwood, Richland, Saluda, and Sumter, had the highest number of checkouts per 100,000 people (Figure 4). Aiken, Anderson, Greenwood, Horry, Lexington, Richland, and Sumter had the highest number of renewals (Figure 2). Eight counties, including Aiken, Anderson, Greenwood, Lee, Richland, Saluda, Sumter, and Williamsburg, had the highest number of renewals per 100,000 people (Figure 5).

The northeast and southwest regions of South Carolina were regions of lower usage. Eight counties, Allendale, Barnwell, Clarendon, Dillon, Edgefield, Jasper, Marlboro, and McCormick, had never borrowed materials from the library (Figure 1). In addition to the counties that had never borrowed materials, Laurens, Marion, and Orangeburg had not renewed library materials (Figure 2). The following fourteen counties also had low per capita checkout rates: Beaufort, Berkeley, Cherokee, Chesterfield, Colleton, Dorchester, Florence, Hampton, Lancaster, Lee, Marion, Newberry, Orangeburg, and Spartanburg (Figure 4). Concerning the counties that had renewed library items, the following ten counties had the lowest number of renewals per 100,000 people: Abbeville, Beaufort, Berkeley, Cherokee, Chester, Chesterfield, Fairfield, Hampton, Spartanburg, and Union (Figure 5).

The maps accurately reflect the use of print and audiovisual materials, but the library usage data does not account for information photocopied or web article links emailed to patrons. Though these services are readily available at the library, the fact that items such as photocopies and web article links cannot be traced back to individual patrons required their exclusion from the data-gathering process, and may potentially threaten the validity of the data obtained.

Other potential confounding variables not discussed in this paper include the number of patrons by county, those patrons who moved between counties or out of the state altogether; the driving distance required of patrons to use

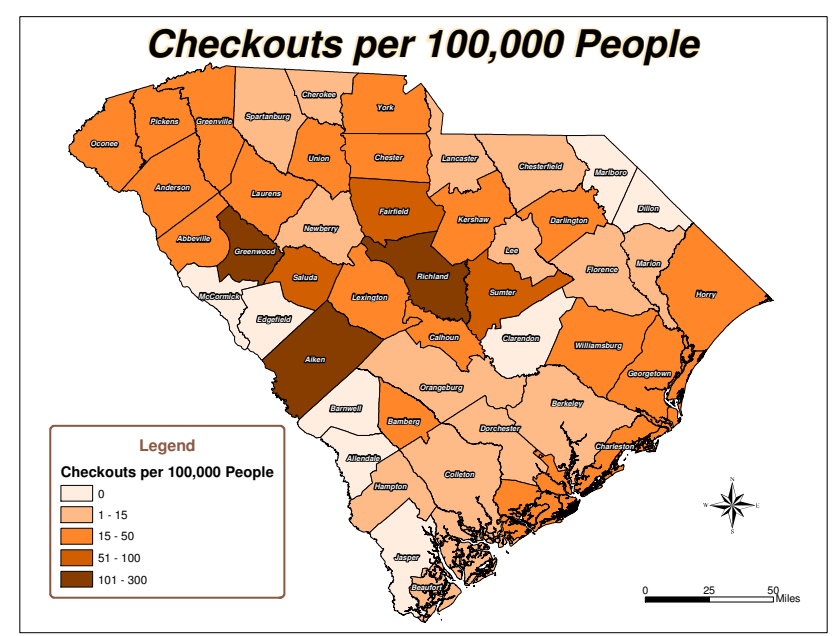

\section{Figure 4}

Checkouts Per 100,000 People. 


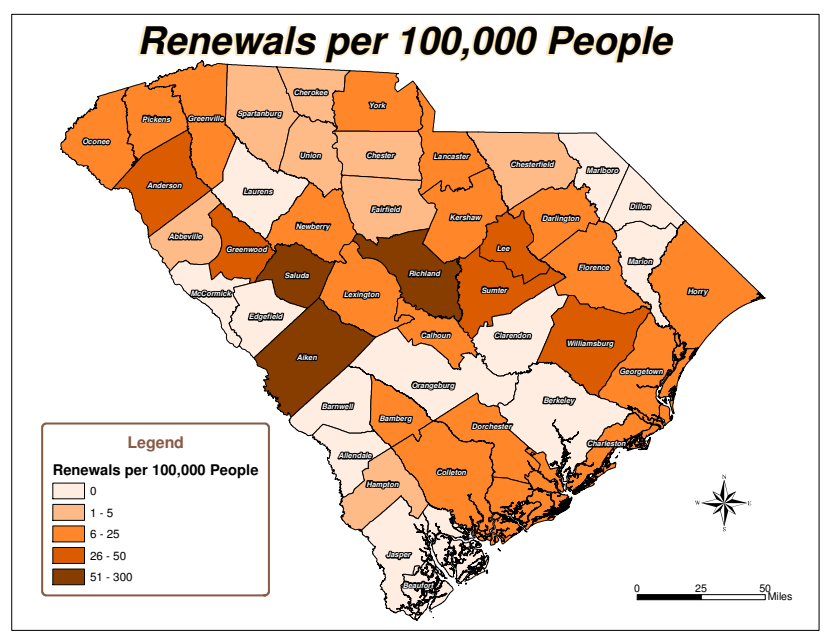

Figure 5

Renewals Per 100,000 People.

the library in person; the relative proportion of special needs individuals by county; and, the relative availability of disability-related materials by public library and each public library's respective location within its county. While it would have been most useful to have a shared legend for comparison purposes, the extreme data distributions required that each map's quintiles be adjusted accordingly.

\section{Conclusion}

Using GIS to identify low usage areas of library materials is an effective means for identifying future marketing areas. The visual representation of the data made identification of low usage regions easier than using a standalone database with no visual-spatial component. Not only could low usage counties be identified, regions of low checkouts and renewals were also evident in the maps. These maps may now be used to communicate visually to the Center for Disability Resources director the need for increased funding for outreach efforts aimed specifically at low usage counties. Moreover, in addition to suggesting counties where new patrons may be solicited, the maps illustrate, literally, those areas with existing users who should continually be encouraged, via marketing and outreach efforts, to take advantage of the library's valuable resources.

There are a number of ways to market the library to new patrons based on location. Since each county has a Disability and Special Needs (DSN) Board that serves individuals with disabilities and their families, CDR Librarians could offer to give presentations to DSN Boards in low usage counties. CDR Librarians may also identify support groups and occupations designed to help individuals with disabilities and their families in these low use areas. Local libraries and relevant businesses could house CDR Library pamphlets or a temporary display about the collection. Future studies using ArcView GIS may determine how successful such marketing efforts are at attracting new patrons to various library collections and services.

\section{Abbreviations \\ $\mathrm{CDR}=$ Center for Disability Resources \\ DSN = Disability and Special Needs \\ GIS = Geographic Information Systems}

\section{Competing interests}

The author(s) declare that they have no competing interests.

\section{Authors' contributions}

RPM created the database, the maps, and drafted the manuscript. SPW managed patron usage statistics and identified patron counties. SPW edited and revised the manuscript. All authors read and approved the final manuscript.

\section{Acknowledgements}

The authors gratefully thank Jeff Anderson from Woolpert, Inc. for his GIS technical support.

\section{References}

I. South Carolina Disability Data Table from the 2004 American Community Survey [http://www.pascenter.org/ state based stats/state statistics 2004.php?state=southcarolina]

2. IDEA: Digest of Education Statistics Tables and Figures. Table 54. [http://nces.ed.gov/programs/digest/d03/tables/ dt054.asp].

3. LaRue EM: Using GIS to establish a public library consumer healthcollection. Biomedical Digital Libraries 2004, I:3. (I8 November 2004)

4. Kinikin J: Applying Geographic Information Systems to the Weber County Library. Information Technology and Libraries 2004, 3:102-107.

5. Xia J: Using GIS to Measure In-Library Book-Use Behavior. Information Technology and Libraries 2004, 4: 184-191.

6. United States Postal Service Zip Code Lookup [http:// zip4.usps.com/zip4/welcome.jsp]

Publish with Bio Med Central and every scientist can read your work free of charge

"BioMed Central will be the most significant development for disseminating the results of biomedical research in our lifetime. "

Sir Paul Nurse, Cancer Research UK

Your research papers will be:

- available free of charge to the entire biomedical community

- peer reviewed and published immediately upon acceptance

- cited in PubMed and archived on PubMed Central

- yours - you keep the copyright

Submit your manuscript here:

http://www.biomedcentral.com/info/publishing_adv.asp 
7. Annual Estimates of the Population for Counties of South Carolina: April I, 2000 to July I, 2003 [http://www.census.gov/ popest/counties/tables/CO-EST2003-0I-45.pdf]

Publish with Bio Med Central and every scientist can read your work free of charge

"BioMed Central will be the most significant development for disseminating the results of biomedical research in our lifetime. " Sir Paul Nurse, Cancer Research UK

Your research papers will be:

- available free of charge to the entire biomedical community

- peer reviewed and published immediately upon acceptance

- cited in PubMed and archived on PubMed Central

- yours - you keep the copyright

Submit your manuscript here:

http://www.biomedcentral.com/info/publishing_adv.asp 